\title{
HEATS OF COMBUSTION OF FIVE ALKYL PHENYL KETONES
}

\author{
M. Colomina, G. Latorre and R. Perez-Ossorio \\ Instituto de Química Fisica, Serrano 119, Madrid, Spain
}

As a contribution to the determination of fundamental thermodynamic properties of pure organic compounds a group of alkyl phenyl ketones has been selected for study.

\section{METHOD AND APPARATUS}

The heats of combustion of the compounds were determined by burning the liquid alkyl phenyl ketones in oxygen in a bomb, at constant volume. The procedure is similar to that described by Prosen ${ }^{1}$, followed by us in other studies ${ }^{2}$.

\section{Materials}

The methyl, ethyl and propyl phenyl ketones were commercial products. The iso- or tert-butyl phenyl ketones were synthesized in this laboratory. The five ketones were purified first by vacuum distillation, in a column of fifty theoretical plates, and then by fractional melting, with an apparatus developed in this laboratory from a simpler device described by Nicholson ${ }^{3}$. The qualitative control of purity by heating curves, following the method of Skau, showed that these compounds were very pure.

\section{EXPERIMENTAL RESULTS}

The results of a series of calibration experiments using N.B.S. standard benzoic acid are given in Table 1. The tabulated data were obtained as in the following paper ${ }^{2}$. The values of $q_{\mathrm{i}}$ were obtained from separate ignition experiments in which a standard mass of $8.30 \mathrm{mg}$ of iron was burned (standard $q_{\mathrm{i}}=62 \mathrm{~J}$ ).

Table 1. Data from calibrating experiments with benzoic acid

\begin{tabular}{c|c|c|c|c|c|c|c}
\hline $\begin{array}{c}\text { Expt } \\
\text { No. }\end{array}$ & $\begin{array}{c}\text { Mass of } \\
\text { benzoic } \\
\text { acid }(\mathrm{g})\end{array}$ & $\begin{array}{c}\Delta e_{1} \\
(\mathrm{~J} / \Omega)\end{array}$ & $\begin{array}{c}\Delta R_{\mathrm{c}} \\
(\Omega)\end{array}$ & $\begin{array}{c}q_{\mathrm{I}} \\
(\mathrm{J})\end{array}$ & $\begin{array}{c}q_{\mathrm{n}} \\
(\mathrm{J})\end{array}$ & $\begin{array}{c}E_{\mathrm{S}} \\
(\mathrm{J} / \Omega)\end{array}$ & $\begin{array}{c}\text { Deviation } \\
\text { from mean } \\
(\mathrm{J} / \Omega)\end{array}$ \\
\hline 1 & $1 \cdot 59823$ & $19 \cdot 2$ & $0 \cdot 299388$ & $61 \cdot 7$ & $1 \cdot 7$ & 141294 & -25 \\
2 & $1 \cdot 59794$ & $19 \cdot 2$ & $0 \cdot 299233$ & $62 \cdot 0$ & $1 \cdot 9$ & 141343 & +25 \\
3 & 1.59979 & $19 \cdot 2$ & $0 \cdot 299624$ & $61 \cdot 8$ & $2 \cdot 0$ & 141322 & +3 \\
4 & $1 \cdot 60135$ & $19 \cdot 2$ & $0 \cdot 299945$ & $63 \cdot 8$ & $1 \cdot 9$ & 141313 & -6 \\
5 & $1 \cdot 59822$ & $19 \cdot 2$ & $0 \cdot 299342$ & $62 \cdot 4$ & $2 \cdot 7$ & 141320 & +1 \\
\hline
\end{tabular}




\section{GOLOMINA, G. LATORRE AND R. PEREZ-OSSORIO}

In Table 2 the results of five series of combustion experiments with the alkyl phenyl ketones are given. These data were obtained as in the following paper ${ }^{2}$.

In Table 3 the heats of combustion and formation of the five alkyl phenyl ketones are given. These data and their uncertainties were computed as in the following paper ${ }^{2}$.

\section{GONCLUSIONS}

On comparing the heats of combustion of the alkyl phenyl ketones with the corresponding values for the liquid alkylbenzenes, reported by Prosen, Johnson and Rossini ${ }^{4}$, the following conclusions can be drawn.

Table 2. Data from combustion experiments with alkyl phenyl ketones

\begin{tabular}{c|c|c|c|c|c|c|c|c}
\hline $\begin{array}{c}\text { Expt. } \\
\text { No. }\end{array}$ & $\begin{array}{c}\text { Mass of } \\
\text { carbon } \\
\text { dioxide } \\
(\mathrm{g})\end{array}$ & $\begin{array}{c}\Delta e_{2} \\
(\mathrm{~J} / \Omega)\end{array}$ & $\begin{array}{l}\Delta R_{\mathrm{c}} \\
(\Omega)\end{array}$ & $\begin{array}{c}Q_{28^{\circ} \mathrm{C}} \\
(\mathrm{J})\end{array}$ & $\begin{array}{c}q_{\mathrm{i}} \\
(\mathrm{J})\end{array}$ & $\begin{array}{c}q_{\mathrm{n}} \\
(\mathrm{J})\end{array}$ & $\begin{array}{c}-\Delta E_{\mathrm{B}} \\
\left(\begin{array}{l}\left.28^{\circ} \mathrm{C}\right) \\
(\mathrm{J} / \mathrm{g} \text { of } \\
\left.\mathrm{CO}_{2}\right)\end{array}\right.\end{array}$ & $\begin{array}{c}\text { Mean value } \\
-\Delta E_{\mathrm{B}} \\
(\mathrm{J} / \mathrm{g} \text { of } \\
\left.\mathrm{CO}_{2}\right)\end{array}$ \\
\hline \\
\hline
\end{tabular}

\begin{tabular}{|c|c|c|c|c|c|c|c|c|}
\hline \multicolumn{9}{|c|}{ Ethyl phenyl ketone } \\
\hline $\begin{array}{l}1 \\
2 \\
3 \\
4\end{array}$ & $\begin{array}{l}3 \cdot 51073 \\
3 \cdot 51625 \\
3 \cdot 51463 \\
3 \cdot 51244\end{array}$ & $\begin{array}{l}23 \cdot 4 \\
23 \cdot 4 \\
23 \cdot 4 \\
23 \cdot 4\end{array}$ & $\begin{array}{l}0 \cdot 301502 \\
0 \cdot 301935 \\
0 \cdot 301895 \\
0 \cdot 301670\end{array}$ & $\begin{array}{l}42615 \cdot 1 \\
42676 \cdot 3 \\
42670 \cdot 6 \\
42638 \cdot 8\end{array}$ & $\begin{array}{l}62 \cdot 3 \\
61 \cdot 4 \\
61 \cdot 9 \\
64 \cdot 0\end{array}$ & $\begin{array}{l}2 \cdot 5 \\
2 \cdot 3 \\
2 \cdot 9 \\
2 \cdot 1\end{array}$ & $\begin{array}{l}12120 \cdot 1 \\
12118 \cdot 7 \\
12122 \cdot 4 \\
12120 \cdot 6\end{array}$ & $\begin{array}{r}12120.5 \\
\pm 0.8\end{array}$ \\
\hline \multicolumn{9}{|c|}{ Propyl phenyl ketone } \\
\hline $\begin{array}{l}1 \\
2 \\
3\end{array}$ & $\begin{array}{l}3 \cdot 41445 \\
3 \cdot 41632 \\
3 \cdot 41379\end{array}$ & $\begin{array}{l}22 \cdot 6 \\
22 \cdot 7 \\
22 \cdot 6\end{array}$ & $\begin{array}{l}0 \cdot 299881 \\
0 \cdot 300023 \\
0 \cdot 299922\end{array}$ & $\begin{array}{l}42386 \cdot 8 \\
42406 \cdot 9 \\
42392 \cdot 6\end{array}$ & $\begin{array}{l}47 \cdot 0 \\
50 \cdot 3 \\
50 \cdot 4\end{array}$ & $\begin{array}{l}2 \cdot 1 \\
2 \cdot 1 \\
2 \cdot 0\end{array}$ & $\begin{array}{l}12399 \cdot 6 \\
12397 \cdot 7 \\
12402 \cdot 7\end{array}$ & $\begin{array}{r}12400 \cdot 0 \\
\pm 1.5\end{array}$ \\
\hline \multicolumn{9}{|c|}{ Isobutyl phenyl ketone } \\
\hline $\begin{array}{l}1 \\
2 \\
3 \\
4 \\
5\end{array}$ & $\begin{array}{l}3 \cdot 33339 \\
3 \cdot 34221 \\
3 \cdot 34344 \\
3 \cdot 33878 \\
3 \cdot 33968\end{array}$ & $\begin{array}{l}22 \cdot 0 \\
22 \cdot 0 \\
22 \cdot 1 \\
22 \cdot 0 \\
22 \cdot 0\end{array}$ & $\begin{array}{c}0 \cdot 297763 \\
0 \cdot 298664 \\
0 \cdot 298686 \\
0 \cdot 298275 \\
0 \cdot 298421\end{array}$ & $\begin{array}{l}42086 \cdot 1 \\
42213 \cdot 6 \\
42216 \cdot 3 \\
42158 \cdot 5 \\
42180 \cdot 5\end{array}$ & $\begin{array}{l}60 \cdot 9 \\
61 \cdot 9 \\
61 \cdot 1 \\
61 \cdot 2 \\
61 \cdot 5\end{array}$ & $\begin{array}{l}2 \cdot 3 \\
2 \cdot 5 \\
2 \cdot 5 \\
2 \cdot 4 \\
2 \cdot 3\end{array}$ & $\begin{array}{l}12606 \cdot 7 \\
12611 \cdot 2 \\
12607 \cdot 7 \\
12607 \cdot 9 \\
12610 \cdot 6\end{array}$ & $\begin{array}{r}12608.8 \\
\pm 0.9\end{array}$ \\
\hline
\end{tabular}

tert-Butyl phenyl ketone

\begin{tabular}{r|r|r|r|r|r|r|r|r}
\hline 1 & $3 \cdot 42902$ & $22 \cdot 6$ & $0 \cdot 306826$ & $43367 \cdot 2$ & $61 \cdot 7$ & $4 \cdot 2$ & $12627 \cdot 9$ & \\
2 & $3 \cdot 42394$ & $22 \cdot 6$ & $0 \cdot 306458$ & $43315 \cdot 3$ & $61 \cdot 1$ & $3 \cdot 0$ & $12632 \cdot 0$ & $12632 \cdot 3$ \\
3 & $3 \cdot 37080$ & $22 \cdot 2$ & $0 \cdot 301694$ & $42641 \cdot 8$ & $61 \cdot 4$ & $3 \cdot 1$ & $12631 \cdot 2$ & $\pm 2 \cdot 1$ \\
4 & $3 \cdot 36889$ & $22 \cdot 2$ & $0 \cdot 301686$ & $42640 \cdot 7$ & $62 \cdot 6$ & $2 \cdot 3$ & $12637 \cdot 9$ & \\
\hline
\end{tabular}


HEATS OF COMBUSTION OF FIVE ALKYL PHENYL KETONES

Table 3. Heats of combustion and formation of alkyl phenyl ketones

\begin{tabular}{|c|c|c|c|c|c|c|}
\hline $\begin{array}{c}\text { Compound } \\
(\text { liq. })\end{array}$ & $\begin{array}{c}-\Delta E_{\mathrm{B}} \\
28^{\circ} \mathrm{C} \\
(\mathrm{kJ} / \mathrm{mole})\end{array}$ & $\begin{array}{c}-\Delta E^{\circ} \\
28^{\circ} \mathrm{C} \\
(\mathrm{kJ} / \mathrm{mole})\end{array}$ & $\begin{array}{c}-\Delta H_{\mathrm{c}}^{\circ} \\
28^{\circ} \mathrm{C} \\
(\mathrm{kJ} / \text { mole })\end{array}$ & $\begin{array}{r}\overline{25} \\
(\mathrm{~kJ} / \mathrm{mole})\end{array}$ & $\begin{array}{l}H_{\mathrm{c}}^{\mathrm{o}} \\
\mathrm{C}^{(\mathrm{kcal} /} \\
\text { mole })\end{array}$ & $\begin{array}{l}\Delta H_{\mathrm{f}}^{\circ} \\
25^{\circ} \mathrm{C} \\
\text { (kcal/ } \\
\text { mole) }\end{array}$ \\
\hline Methyl phenyl ketone & $\begin{array}{r}4147.39 \\
\pm 0.89\end{array}$ & $\begin{array}{r}4144.95 \\
+0.89\end{array}$ & $\begin{array}{r}4148.71 \\
+0.89\end{array}$ & $\begin{array}{r}4148.95 \\
+0.89\end{array}$ & $\begin{array}{r}991.62 \\
+0.21\end{array}$ & $\begin{array}{r}-34.06 \\
+0.25\end{array}$ \\
\hline Ethyl phenyl ketone & $\begin{array}{r}4800.90 \\
+\quad 1.05\end{array}$ & $\begin{array}{r}4798.28 \\
+\quad 1.05\end{array}$ & $\begin{array}{r}4803.29 \\
+\quad 1.05\end{array}$ & $\begin{array}{r}4803.66 \\
+\quad 1.05\end{array}$ & $\begin{array}{r}1148 \cdot 10 \\
+\quad 0.25\end{array}$ & $\begin{array}{r}-39.95 \\
+\quad 0.30\end{array}$ \\
\hline Propyl phenyl ketone & $\begin{array}{r}5457.36 \\
+\quad 1.69\end{array}$ & $\begin{array}{r}5454.66 \\
+\quad 1.69\end{array}$ & $\begin{array}{r}5460.83 \\
+\quad 1.69\end{array}$ & $\begin{array}{r}5461.32 \\
+\quad 1.69\end{array}$ & $\begin{array}{r}1305.29 \\
+\quad 0.40\end{array}$ & $\begin{array}{r}-45.13 \\
+\quad 0.45\end{array}$ \\
\hline Isobutyl phenyl ketone & $\begin{array}{r}6104.19 \\
+\quad 1.39\end{array}$ & $\begin{array}{r}6101.23 \\
+\quad 1.39\end{array}$ & $\begin{array}{r}6108.74 \\
+\quad 1.39\end{array}$ & $\begin{array}{r}6109.36 \\
+\quad 1.39\end{array}$ & $\begin{array}{r}1460.17 \\
+0.33\end{array}$ & $\begin{array}{r}+52.62 \\
+0.39\end{array}$ \\
\hline tert-Butyl phenyl ketone & $\begin{array}{r}6115 \cdot 54 \\
\pm 2 \cdot 26\end{array}$ & $\begin{array}{r}6112.57 \\
\pm 2.26\end{array}$ & $\begin{array}{r}6120.09 \\
\pm 2.26\end{array}$ & $\begin{array}{r}6120 \cdot 70 \\
\pm 2 \cdot 26\end{array}$ & $\begin{array}{r}1462.88 \\
\pm 0.54\end{array}$ & $\begin{array}{r}-49.91 \\
\pm 0.60\end{array}$ \\
\hline
\end{tabular}

(a) The increments in the heats of combustion per $\mathrm{CH}_{2}$ group added to the chain in the liquids methyl phenyl ketone and methylbenzene have the same value of $156.5 \mathrm{kcal} / \mathrm{mole}$. This identity of increments in the heats of combustion per $\mathrm{CH}_{2}$ group added to the chain is again reproduced in the liquids propyl phenyl ketone and propylbenzene with the same value of $154.9 \mathrm{kcal} / \mathrm{mole}$.

(b) The increments in the heats of combustion per $\mathrm{CH}_{2}$ group added to the chain in the liquids ethyl phenyl ketone and ethylbenzene have an unexpected difference of $1 \mathrm{kcal} / \mathrm{mole}$ : $157 \cdot 2 \mathrm{kcal} / \mathrm{mole}$ and $156 \cdot 2 \mathrm{kcal} / \mathrm{mole}$, respectively.

(c) There is an important difference between the heats of isomerization for the liquid state at $25^{\circ} \mathrm{C}$ of the following reactions:

Isobutyl phenyl ketone $\longrightarrow$ tert-Butyl phenyl ketone $-2.7 \mathrm{kcal} / \mathrm{mole}$

$$
\text { Isobutylbenzene } \quad \longrightarrow \text { tert-Butyl benzene } \quad+0.2 \mathrm{kcal} / \mathrm{mole}
$$

Finally, from the heat of combustion of benzyl methyl ketone, measured by Springall and Nicholson ${ }^{5,3}$, and our experimental results, the heat of isomerization of the following reaction for the liquid state at $25^{\circ} \mathrm{G}$ has been computed:

Ethyl phenyl ketone $\longrightarrow$ Benzyl methyl ketone $-3.6 \mathrm{kcal} / \mathrm{mole}$

\section{References}

1 E. J. Prosen, Chap. 6 in Experimental Thermochemistry, Ed. F. D. Rossini, Interscience, New York (1956)

${ }^{2}$ M. Colomina, R. Pérez-Ossorio and M. L. Boned. " Thermochemical investigations on methyl-substituted benzoic acids: I. Toluic acids". This Symposium, Pure and Appl. Chem., 2, No. 1-2, 137 (1961)

${ }^{3}$ G. R. Nicholson, M. Szwarc and J. W. Taylor. 7. Chem. Soc., 1954, 2767

4 E. J. Prosen, W. H. Johnson and F. D. Rossini. J. Research Nat Bur. Standards, 36, 455 (1946)

${ }^{5}$ H. D. Springall and R. T. White. J. Chem. Soc., 1954, 2764 